\title{
Vibrational Fatigue and Structural Dynamics for Harmonic and Random Loads
}

\author{
Martin Česnik - Janko Slavič* \\ University of Ljubljana, Faculty of Mechanical Engineering, Slovenia
}

\begin{abstract}
The presented study experimentally and theoretically researches vibrational fatigue of an aluminum-alloy specimen for harmonic and random loads. The main aim of this study is to determine the influence of modal parameter changes that occur during the experimental fatigue test, on the correctness of the numerical fatigue life prediction. Firstly, the material's fatigue parameters were obtained with harmonic base excitation of the specimen near its natural frequency. During the harmonic fatigue testing the changes in the specimen's natural frequency and damping loss factor were monitored as the fatigue damage was accumulated at the fatigue zone. Secondly, with a validated numerical model of the specimen the stress transmissibility was obtained for the case of the random-vibration base excitation. Finally, by respecting the stress response along with the experimentally obtained material fatigue parameters the vibration fatigue life was estimated for the case of the random vibration load. The numerically predicted fatigue life was compared to the experimental results, obtained with the electro-dynamic shaker. From this comparison the influence of the damping-loss-factor changes on the calculated fatigue life was clearly shown. For the case of the observed specimen, the damping loss factor included in the fatigue life estimation should be increased by more than 100 percent to give reliable prediction of the fatigue life. The presented research shows new possibilities and critical aspects in the area of accurate highcycle vibration fatigue life-estimation of dynamic structures.
\end{abstract}

Keywords: structural dynamics, vibrational fatigue, fatigue testing, harmonic excitation, random excitation, frequency-domain counting methods

\section{O INTRODUCTION}

The fatigue failure can occur even if the structure is loaded with a low-amplitude cyclic loads when the load is applied for a longer period of time. Each cycle represents a certain amount of fatigue damage that is being accumulated by the structure; when the accumulated fatigue damage at the given point on the structure reaches a certain value (usually defined as unity), the fatigue failure occurs [1]. Stress cycles that lead to the fatigue failure are a result of external loads of the structure (e.g. mechanical, temperature, vibrational load). The type of fatigue failure due to the vibrational load of the structure is known as vibrational fatigue and has been a subject of several research studies in recent years. Vibrational fatigue can be studied for harmonic or random vibration loads. In both cases the stress load leading to the fatigue failure occurs due to the structure's own dynamic response [2] to the dynamic base-excitation, [3] and [4].

Whenever vibrational fatigue occurs it is always accompanied with changes in the natural frequency and damping loss factor. This phenomenon can be utilized to predict the fatigue failure, [5] to [7]. However, when obtaining the fatigue parameters by applying harmonic vibration load, the changes in natural frequency and damping as a result of the increased local nonlinearities [8], lead to the varying amplitude of the stress load cycles and consequentially to the incorrect identification of the fatigue parameters. The first part of this study deals with the experimental setup and a real-time control of an accelerated vibration fatigue test with harmonic excitation [9], that in a short time accurately obtains fatigue parameters and simultaneously monitors the changes in the natural frequency and damping loss factor.

When the fatigue parameters are known one can predict the fatigue life of the structure exposed to a random-signal vibrational load using the power spectral density of the structure's stress response, [10] and [11]. Recently, several research studies have been done in the area of the vibrational fatigue under a random base-excitation. Pagnacco et al. [12] optimised a thickness distribution of a plate structure to prolong the structure's fatigue life when exposed to the random vibration. Furthermore, Paulus and Dasgupta [13] proposed a semi-empirical model for fatigue life estimation for the case of the random vibration load. In their study they modeled a cantilevered beam as a single-degree-of-freedom (SDOF) lumped-parameter system and included the beam's natural frequency shift due to the damage accumulation into the fatigue life calculation. Han et al. [14] performed the vibration fatigue analysis of a spot-welded structure with an iterative approach. Research studies relating the fatigue parameters obtained with harmonic excitation to the fatigue life under the random base-excitation were presented by $\mathrm{Chen}$ et al. [15] and Yu et al. [16], where the ball grid array (BGA) solder joints were the 
subject of analysis. In this study, the second part is focused on the estimation and experimental validation of the fatigue life prediction of a linear Y-shaped structure under a random vibration loading, where the fatigue parameters obtained with the harmonic fatigue tests are used. Furthermore, by comparing numerical and experimental results it is clearly shown that the changes of the modal parameters must be adequately considered in order to obtain reliable fatigue life estimation.

This manuscript is organized as follows. In Section 1 the theoretical background is given, concerning the specimen's response to the base excitation and the identification of instantaneous natural frequency and damping loss factor. Additionally, some fundamentals of the fatigue life calculation in the frequency domain are given. In Section 2 the experimental setup of the fatigue test with the harmonic excitation is presented along with the obtained experimental results, needed to obtain $\sigma-N$ curve of the material in question. In Section 3 the numerical and experimental results for a fatigue test with random base-excitation are shown and compared. In Section 4 the conclusions are given.

\section{THEORETICAL BACKGROUND}

When a dynamic system is excited in the resonant area dynamic response amplification can be observed. By taking advantage of this response amplification it is possible to achieve high stresses in the specimen by applying relatively small excitation displacements, which makes an electro-dynamic shaker suitable for performing fatigue tests. The idea of the accelerated vibrational fatigue test is based on the dynamic response of the specimen; therefore, it is necessary to research the dynamic properties of the specimen before the actual fatigue test. Furthermore, the specimen's dynamic response to the random baseexcitation represents a basis for the estimation of the fatigue life with frequency-domain counting methods.

\subsection{Specimen's Dynamic Response to the Harmonic Excitation}

The dynamic response of the specimen depends on its modal properties; i.e., natural frequencies, mode shapes and damping loss factors. The natural frequencies and mode shapes are easily obtained using the finite-element method and should be determined in the stage of the experiment design. For the purpose of the vibration fatigue test the natural frequency, near which the specimen is excited, must be well separated from the remaining natural frequencies and must be within the shaker's frequency range. Additionally, the excited mode shape of interest must be excitable with a translational movement in the axis of the shaker. However, by exciting the specimen in different mode shapes it is possible to achieve different stress states with a single specimen geometry.

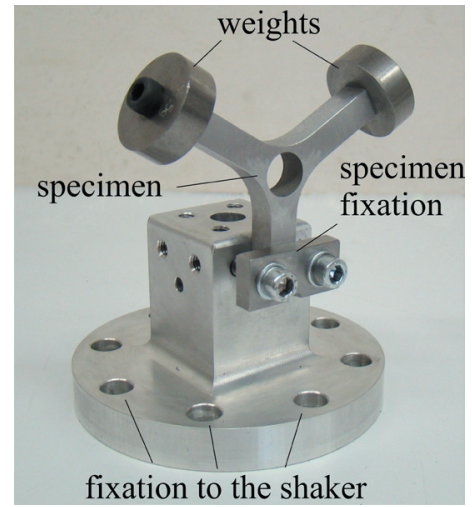

Fig. 1. Fixed Y-shaped specimen

In this research the Y-shaped specimen shown in Fig. 1 is used [9]. The main three beams are arranged at $120^{\circ}$ around the main axis and have a rectangular cross-section of $10 \times 10 \mathrm{~mm}$. The Y-shaped specimen was made from aluminum alloy A-S8U3 by casting and with a surface finish performed by milling. The fatigue zone was additionally fine-ground in order to remove any scratches that could cause the premature start of an initial crack. Two additional features are included in the Y-shaped design: steel dead-weights of mass $52.5 \mathrm{~g}$ and a round hole through the main axis. These two features are used to adjust the initial natural frequency and to position the fatigue zone on a suitable surface, respectively.

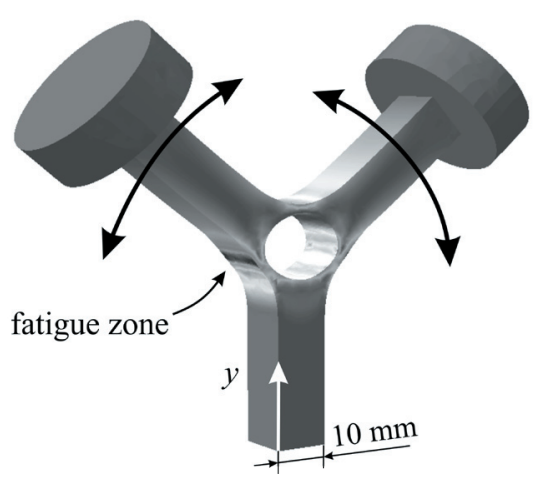

Fig. 2. Specimen's fourth-mode shape, $\sigma_{e q}$

When performing the numerical analysis of the proposed specimen the material parameters of aluminum were used, e.g. density of $\rho=2710 \mathrm{~kg} / \mathrm{m}^{3}$ 
and Young modulus of $E=70 \mathrm{MPa}$. By evaluating the dynamic response of the Y-shaped specimen the fourth-mode shape at $\omega_{1}=755 \mathrm{~Hz}$ (Fig. 2) was recognized as the most suitable for the near-resonance fatigue test. For the sake of simplicity the fourth natural frequency is in this manuscript denoted as $\omega_{1}$ rather than $\omega_{4}$.

In order to appropriately excite the Y-shaped specimen with harmonic signal and simultaneously maintain constant stress amplitude some theoretical background regarding the system's response to harmonic base excitation must be given. Additionally, during the accelerated fatigue test the changes to the modal parameters of the specimen occur [6] and [7], which consequently alter the specimen's response and must also be taken into the account in theoretical discussion. The following discussion is applied to the basic outline of the accelerated vibration fatigue experiment, illustrated in Fig. 3, and summarizes the methods, presented in [9].

Continuous structures, such as a Y-shaped specimen, are generally described as multi-degree-offreedom (MDOF) systems; in the case of a kinematic excitation and a hysteretic damping mechanism the equilibrium equation can be written as [17]:

$$
\mathbf{M} \ddot{\mathbf{x}}^{T}+\mathrm{i} \mathbf{D} \mathbf{x}^{T}+\mathbf{K} \mathbf{x}^{T}=\mathbf{0} .
$$

Here $\mathbf{x}^{T}$ denotes the total displacement vector and $\mathbf{M}, \mathbf{D}$ and $\mathbf{K}$ are the mass, damping and stiffness matrices, respectively. For the case of kinematic excitation [4] $\mathbf{x}^{T}$ can be written as:

$$
\mathbf{x}^{T}(t)=\mathbf{x}(t)+\mathfrak{l} y(t),
$$

where $\mathbf{x}(t)$ is a vector of relative response displacements, $\mathbf{l}$ is the geometry-related column vector and $y(t)$ is the base-excitation signal.
According to the Eqs. (1) and (2) the system's relative response $\bar{X}_{a}$ of the $a^{\text {th }}$ degree-of- freedom in the case of the base excitation $y(t)=Y \sin (\omega t)$ is deduced as [17]:

$$
\begin{aligned}
\bar{X}_{a} & =\mathbf{m}_{a} \mathbf{\imath} \omega^{2} Y \alpha_{X_{a}}(\omega)= \\
& =\mathbf{m}_{a} \mathbf{\imath} \omega^{2} Y \sum_{r=1}^{N} \frac{{ }_{r} A_{X_{a}}}{\omega_{r}^{2}-\omega^{2}+\mathrm{i} \eta_{r} \omega_{r}^{2}},
\end{aligned}
$$

where $\mathbf{m}_{a}$ denotes the $a^{\text {th }}$ row in the mass matrix $\mathbf{M}$ and $\alpha_{X_{a}}(\omega)$ is the receptance function for the $a^{\text {th }}$ degree of freedom. Additionally, ${ }_{r} A_{X_{a}}$ represents the mass-normalized modal constant for the $a^{\text {th }}$ degree-of-freedom, $\omega_{r}$ is the $r^{\text {th }}$ natural frequency and $\eta_{r}$ is the hysteretic damping loss factor for the $r^{\text {th }}$ mode shape. Here it should be noted that since the excitation and response are single harmonics with a known frequency $\omega$ the relations $\ddot{x}(t)=-\omega^{2} \cdot x(t)$ and $\ddot{y}(t)=-\omega^{2} \cdot y(t)$ between the measured accelerations and displacements are valid.

If a dynamic system is observed when excited near the $p^{\text {th }}$ natural frequency the ratio $\bar{X}_{a} / Y$ can be approximated as [17]:

$$
\frac{\bar{X}_{a}}{Y} \approx \mathbf{m}_{a} \boldsymbol{\imath} \omega^{2}\left(\frac{{ }_{p} A_{X_{a}}}{\omega_{p}^{2}-\omega^{2}+\mathrm{i} \eta_{p} \omega_{p}^{2}}+B_{X_{a}}\right),
$$

where $B_{X_{a}}$ is a constant complex quantity, in which the contribution of the remaining modes $r \neq p$ is accounted for.

A similar deduction to that in Eq. (4) can be made for the frequency response of the measured principal stress $\overline{\sigma_{1}}$ to the base excitation $y(t)$. As shown by Česnik et al. [9], by comparing stress and relative displacement responses to the same base excitation, the relation:

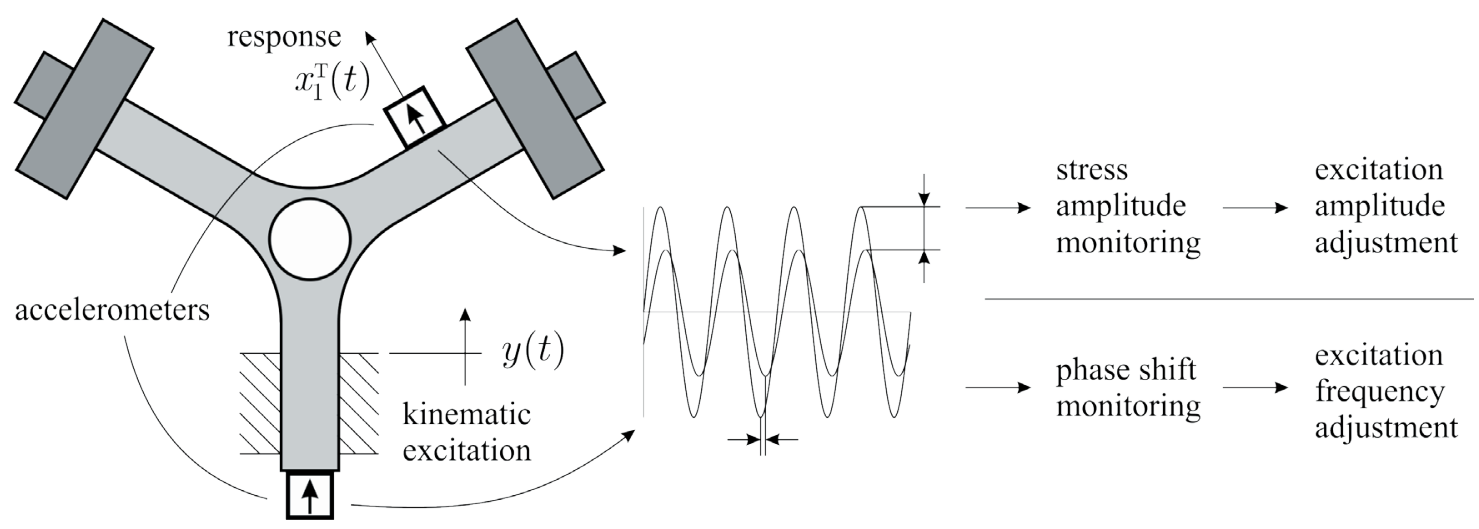

Fig. 3. Experimental setup outline 


$$
\frac{\sigma_{1}}{X_{1}}=\text { const. }
$$

holds in the vicinity of a well-separated natural frequency even when the system's natural frequency and damping loss factor change, as long as the constant phase shift $\phi$ between the excitation and response signal is maintained. This observation enables the indirect monitoring of the principal stress $\sigma_{1}$, using only the measured excitation and response accelerations; however, the ratio $\sigma_{1} / X_{1}$ must be experimentally obtained before the accelerated fatigue test is conducted.

By knowing the constant stress amplitude at the fatigue zone and a number of load cycles until fatigue failure the fatigue test of one Y-shaped specimen directly gives a single point on the $\sigma-N$ diagram. From this point on, the procedure for the calculation of fatigue parameters is identical to the procedure when classical fatigue testing machines are used [1].

\subsection{Simultaneous Identification of the Natural Frequency and Damping Loss Factor}

In order to simultaneously track changes in the natural frequency $\omega_{1}$ and damping loss factor $\eta_{1}$ in the case of the near-resonant excitation a simple identification procedure is presented here, which is based on the linear single-degree-of-freedom (SDOF) assumption [17].

In Eq. (4) it was shown that the response of a base-excited structure can be modelled with the modified receptance:

$$
\alpha_{X_{a}}(\omega)=\frac{\bar{X}_{a}}{Y}=\frac{\omega_{p}^{2} A_{X_{a}}^{\prime}}{\omega_{p}^{2}-\omega^{2}+\mathrm{i} \eta_{p} \omega_{p}^{2}},
$$

where the SDOF assumption is considered with $B_{X}^{\prime}=0$. The receptance is fully defined with known values of the natural frequency $\omega_{p}$, the damping loss factor $\eta_{p}$ and the modal constant ${ }_{p} A_{X_{a}}^{\prime}$ for the $p^{\text {th }}$ mode shape. These values can be easily determined from the experimentally obtained response of the dynamic system using the circle-fitting method, described in [17].

By measuring the dynamic response of the Y-shaped specimen before the accelerated fatigue test, the initial receptance is obtained. As the accelerated vibration fatigue test starts, the natural frequency $\omega_{1}$ and the damping loss factor $\eta_{1}$ begin to change, therefore introducing two new variables into Eq. (6) that must be identified. The value of modal constant ${ }_{p} A_{X_{a}}^{\prime}$ is assumed constant during the fatigue test.

The identification of $\omega_{1}$ and $\eta_{1}$ can be made with a numerical solution of the system of equations:

$$
\begin{gathered}
\frac{X_{1}}{Y}=\left|\alpha_{X_{1}}\left(\omega, \omega_{1}, \eta_{1}\right)\right|, \\
\phi=\arg \left(\alpha_{X_{1}}\left(\omega, \omega_{1}, \eta_{1}\right)\right),
\end{gathered}
$$

where the phase angle $\phi$, relative response $X_{1}$, excitation amplitude $Y$ and excitation frequency $\omega$ are measured during the accelerated fatigue test.

\subsection{Fatigue Life Estimation for the Random Base- Excitation}

Contrary to the simple fatigue life estimation methods for the case of harmonic stress history [1] the estimation of fatigue life for random stress history requires a brief theoretical introduction. Damage estimation for random stress history can be done with time- or frequency-domain methods. In this study only the Tovo-Benasciutti frequency-domain counting method [11] is used, which has shown to be very accurate for a number of different stress profiles [18].

The frequency domain counting methods are based on the frequency stress response of the excited structure. In the case of vibration excitation with a given acceleration power spectral density (PSD) matrix $\mathbf{S}_{\mathrm{aa}}(\omega)$ the stress response PSD matrix is [17]:

$$
\mathbf{S}_{\sigma \sigma}(\omega)=\left|\mathbf{H}_{\sigma \mathbf{a}}(\omega)\right|^{2} \mathbf{S}_{\mathrm{aa}}(\omega)
$$

where $\mathbf{H}_{\sigma \mathrm{a}}(\omega)$ represents the acceleration-to-stress transmissibility matrix of the structure. Stress response PSD is obtained from numerical model, that needs to be build and validated with the actual test specimen. Since the general stress state is multi-axial, the PSD of the equivalent stress $S_{\sigma_{e q}}(\omega)$ is used for the damage intensity estimation as [19]:

$$
S_{\sigma_{e q}}(\omega)=\operatorname{Trace}\left[\mathbf{Q S} \mathbf{S}_{\sigma \sigma}(\omega)\right]
$$

where $\mathbf{Q}$ is constant matrix, defined in [19]. With known specimen's PSD the Tovo-Benasciutti method estimates the damage intensity as:

$$
D=\left(B_{T B}+\left(1-B_{T B}\right) \alpha_{2}^{B_{T B}-1}\right) \alpha_{2} D_{N B} .
$$

Here $D_{N B}$ is a damage intensity for the narrow band stress profile and $B_{T B}$ is a factor, obtained from spectral characteristics of the stress PSD: 


$$
D_{N B}=\alpha_{2} v_{p} C^{-b}\left(\sqrt{2 \lambda_{0}}\right)^{b} \cdot \Gamma\left(1+\frac{b}{2}\right)
$$

$$
\begin{aligned}
& B_{T B}=\frac{\left(\alpha_{1}-\alpha_{2}\right)}{\left(\alpha_{2}-1\right)^{2}} \times \\
& \times\left(1.112\left(1+\alpha_{1} \alpha_{2}-\left(\alpha_{1}+\alpha_{2}\right)\right) \mathrm{e}^{2.11 \alpha_{2}}+\left(\alpha_{1}-\alpha_{2}\right)\right) .
\end{aligned}
$$

In Eqs. (11) to (13) the parameters $\alpha_{1}, \alpha_{2}, v_{p}$ and $\lambda_{0}$ are the statistical characteristics of the $S_{\sigma_{e q}}(\omega)$ (see [11]), $b$ and $C$ are the material fatigue parameters and $\Gamma$ is the gamma function. The estimated fatigue life of the analyzed structure equals to $T_{e s t}=1 / D$.

The numerical analysis of expected fatigue life is generally performed in the following manner. Firstly, the stress response PSD matrix is calculated for the given specimen geometry, boundary conditions and applied random vibration profile. Secondly, the equivalent stress PSD's are obtained for the nodes of interest. Thirdly, the statistical characteristics of the equivalent stress PSD's are calculated. Lastly, by introducing the material's fatigue parameters and statistical characteristics of the stress PSD into damage intensity equation (which depends on the adopted damage-estimation method), the expected fatigue life for a single node is obtained.

\section{FATIGUE TEST WITH HARMONIC EXCITATION}

The first part of the experimental work concerns the harmonic fatigue test. With the series of tests presented here the fatigue parameters for the material A-S8U3 are obtained along with the continuous changes of the natural frequency and damping loss factor. The experimental setup follows the testing methodology presented by Česnik et al. [9].

\subsection{Experiment Setup and Control}

In the presented experimental setup, the Y-shaped specimen is attached with the fixation adapter to the LDS V555 electro-dynamical shaker, Fig 4. To keep the system in the resonant area and to prevent the drop in the response amplitude the excitation frequency $\omega$ must be tracking the decreasing natural frequency $\omega_{1}$ in real-time during the accelerated fatigue test.

For this case of a near-resonant single-harmonic excitation of the MDOF system the tracking of the natural frequency can be performed by maintaining the constant phase shift $\phi$ between the excitation $y(t)$ and the response displacement $x_{1}(t)$, as explained in detail in [9]. The constant phase shift is maintained by adjusting the excitation frequency $\omega$. The stress amplitude is maintained constant by adjusting the excitation amplitude $Y$ according to the indirect measurements of stress amplitude by monitoring the relative displacement $X_{1}$. This is possible since Eq. (5) holds; however, the constant value of $\sigma_{1} / X_{1}$ ratio needs to be determined experimentally before the fatigue test. Therefore, the calibration measurement was performed on the specimens S01 and S02, as shown in Fig. 5. The identified ratio was equal to $\sigma_{1} / X_{1}=3.90 \mathrm{MPa} / \mu \mathrm{m}$.

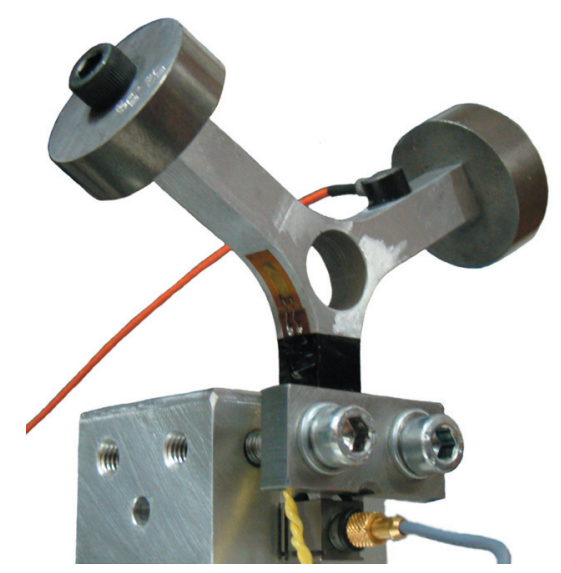

Fig. 4. Calibration experiment setup

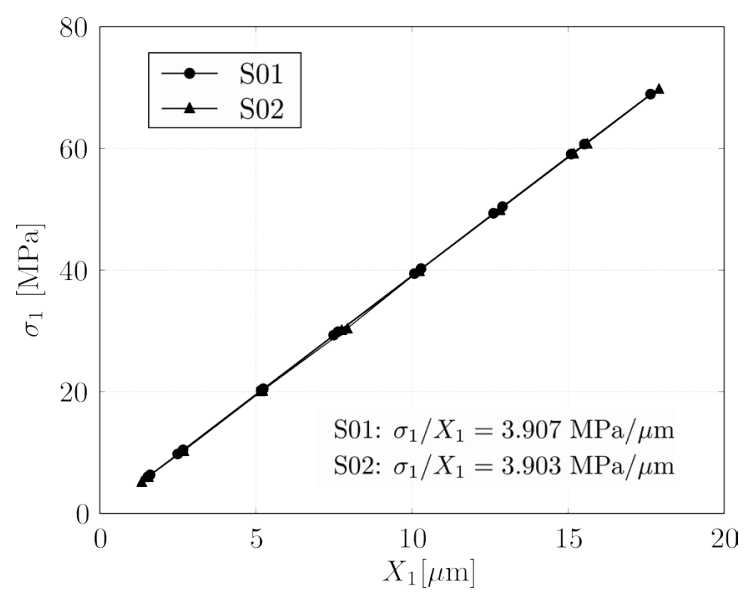

Fig. 5. $\sigma_{1} / X_{1}$ calibration measurement

\subsection{Test Results}

The harmonic fatigue test was performed on nine Y-shaped specimens, denoted with S01-S09. The fatigue failure was recognized when a sudden drop of the natural frequency occurred. The instantaneous natural frequencies, identified with the method presented in Sec. 1.2, are shown in Fig. 6. The changes in the damping loss factors are shown in 
Fig. 7. In order to show the modal parameter changes throughout the fatigue life evenly for all specimens, regardless of their actual fatigue lives, a damage index is used in Figs. 6 and 7. For the case of harmonic load with constant cycle amplitude the damage is defined as Damage $=n / N$ [1], where $n$ and $N$ denote a current number of cycles and a total number of cycles until failure, respectively. The summarized experimental results are listed in Table 1, where $\sigma_{1}$ denotes the principal stress amplitude, $f_{1 \text { init }}$ is the initial natural frequency, $\min \left(\eta_{1}\right)$ is the minimal damping loss factor and $\Delta f_{1}$ is the total natural frequency shift.

Table 1. Experimental data of harmonic fatigue test

\begin{tabular}{cccccc}
\hline Specimen & $\begin{array}{c}\sigma_{1} \\
{[\mathrm{MPa}]}\end{array}$ & $\begin{array}{c}\text { Cycles to failure } \\
N\left(\times 10^{5}\right)\end{array}$ & $\begin{array}{c}f_{\text {1init }} \\
{[\mathrm{Hz}]}\end{array}$ & $\begin{array}{c}\min \left(\eta_{1}\right) \\
\left(\times 10^{4}\right)\end{array}$ & $\begin{array}{c}\Delta f_{1} \\
{[\mathrm{~Hz}]}\end{array}$ \\
\hline S01 & 95 & 33.0 & 758.0 & 3.10 & 31.3 \\
\hline S02 & 105 & 25.0 & 751.6 & 2.85 & 18.3 \\
\hline S03 & 105 & 13.5 & 757.2 & 3.08 & 28.6 \\
\hline S04 & 115 & 9.71 & 757.3 & 4.49 & 21.2 \\
\hline S05 & 115 & 13.1 & 754.3 & 3.73 & 34.8 \\
\hline S06 & 125 & 6.87 & 755.8 & 3.96 & 21.9 \\
\hline S07 & 125 & 7.98 & 755.7 & 4.60 & 22.2 \\
\hline S08 & 135 & 4.87 & 752.3 & 5.37 & 20.9 \\
\hline S09 & 135 & 3.24 & 753.4 & 6.09 & 16.2 \\
\hline
\end{tabular}

Fig. 6 indicates that specimens show different frequency shifts and no value of the frequency shift can be used as a definite value for the indication of the final fatigue failure. At this point it should be mentioned that no significant temperature rise was measured during the fatigue testing; the temperature rise was within $2{ }^{\circ} \mathrm{C}$ and can be addressed to the heat conduction from the shaker's core to the test specimen. Additionally, from Fig. 7 it can be noticed that damping loss factor varied significantly between the tested specimens and was higher for higher loads. This can be addressed to the damping that occurs locally at the fixation of the Y-shaped specimen. Therefore, in the random fatigue test the damping loss factor, used for the numerical prediction of the fatigue life, needs to be identified and considered in the numerical model separately for each tested specimen.

The material's fatigue parameters, fatigue exponent $b$ and fatigue strength $S_{f}$ were calculated with least squares method. The Basquin's equation [1] for the aluminum alloy A-S8U3 is:

$$
\sigma=688.1 N^{-0.170}
$$

and is illustrated in the Fig. 8 along with the $\sigma-N$ experimental results.

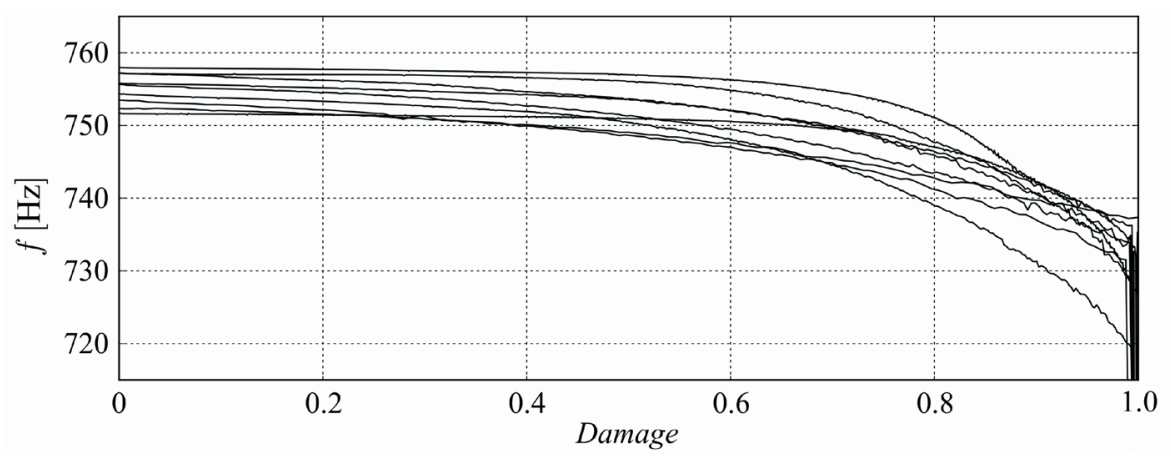

Fig. 6. Identified natural frequencies for samples S01-S09

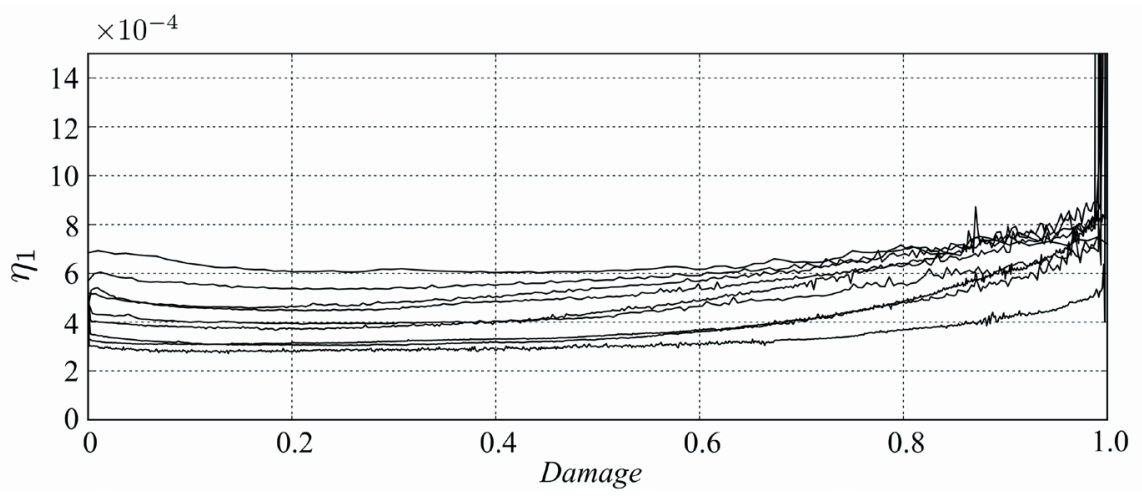

Fig. 7. Identified damping loss factor for samples S01-S09 


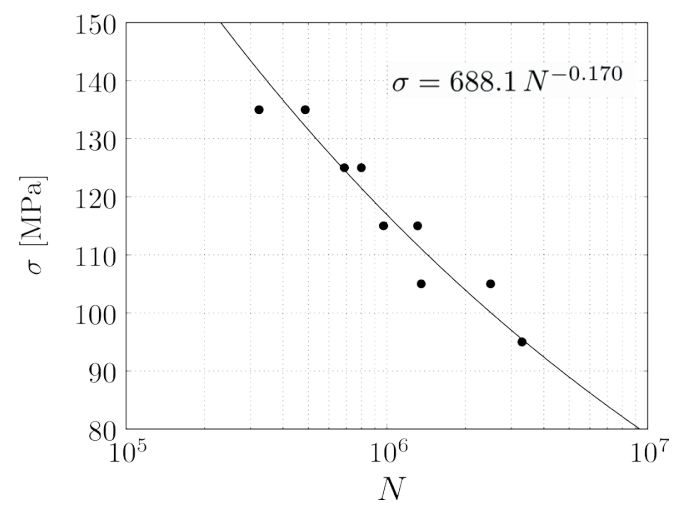

Fig. 8. $\sigma-N$ diagram and corresponding Wöhler curve for harmonic excitation

\section{EXPERIMENT WITH RANDOM EXCITATION AND VIBRATION FATIGUE ANALYSIS}

In this section, the second experiment is presented, where the Y-shaped specimens are excited with the random acceleration profile. Here, a constant random acceleration profile is maintained at the fixation of the specimen during the whole vibration test until the fatigue failure occurs. In this way the conditions during a standard vibration test [20] are reproduced.

Firstly the experimental setup and results are presented. Secondly, a numerical model for the fatigue life estimation is introduced. Finally, the comparison between experimental and predicted fatigue lives of specimens is given.

\subsection{Experiment Setup and Results}

Experimental setup of the fatigue test with the random base-excitation is identical to the setup, illustrated in Fig. 4, except no strain gages were used to monitor stress levels in the fatigue zone. The test was performed on the LSD V875 shaker, the acceleration profiles had constant level of the acceleration PSD in the frequency band of 600 to $800 \mathrm{~Hz}$. The selection of a narrow band random profile in the vicinity of a single natural frequency greatly reduces the influence that the remaining modes have on system's dynamic response and damage accumulation. However, the frequency band of excitation should be wide enough to ensure the appropriate excitation of the observed modeshape even when the drop of natural frequency occurs. The excitation of the specimen was controlled with a Dactron Laser controller. Ten Y-shaped specimens S11 to S20 were tested: at the start of each test the initial transmissibility was measured with very low acceleration profile to obtain initial damping, afterwards the fatigue test was performed. During the fatigue test only the transmissibility magnitude between response and control accelerometer (Fig. 4) was measured from which the changes of natural frequency with time were identified. The results of the experiment are summarized in Table 2.

Table 2. Experimental data of random-excitation fatigue test

\begin{tabular}{cccccc}
\hline $\begin{array}{c}\text { Speci- } \\
\text { men }\end{array}$ & $\begin{array}{c}\text { PSD level } \\
{\left[\left(\mathrm{m} / \mathrm{s}^{2}\right)^{2} / \mathrm{Hz}\right]}\end{array}$ & $\begin{array}{c}\text { Time to failure } \\
\times 10^{2}[\mathrm{~s}]\end{array}$ & $\begin{array}{c}f_{1 \text { init }} \\
{[\mathrm{Hz}]}\end{array}$ & $\begin{array}{c}\eta_{1 \text { init }} \\
\times 10^{4}\end{array}$ & $\begin{array}{c}\Delta f_{1} \\
{[\mathrm{~Hz}]}\end{array}$ \\
\hline $\mathrm{S} 11$ & 0.725 & 391 & 754 & 4.07 & 71.7 \\
\hline $\mathrm{S} 12$ & 0.725 & 316 & 749 & 5.07 & 55.6 \\
\hline $\mathrm{S} 13$ & 1.08 & 65.0 & 752 & 4.31 & 140.4 \\
\hline $\mathrm{S} 14$ & 1.08 & 98.7 & 755 & 4.64 & 43.7 \\
\hline $\mathrm{S} 15$ & 1.60 & 27.4 & 749 & 3.80 & 139.7 \\
\hline $\mathrm{S} 16$ & 1.60 & 18.8 & 750 & 7.10 & 60.8 \\
\hline $\mathrm{S} 17$ & 2.38 & 13.6 & 745 & 5.39 & 75.6 \\
\hline $\mathrm{S} 18$ & 2.38 & 15.2 & 758 & 4.59 & 114.3 \\
\hline $\mathrm{S} 19$ & 5.25 & 3.98 & 754 & 4.87 & 75.9 \\
\hline $\mathrm{S} 20$ & 5.25 & 3.84 & 754 & 5.77 & 62.6 \\
\hline
\end{tabular}

The natural frequency shifts during the randomexcitation fatigue tests are shown in Fig. 9. From it one can observe, that significantly larger natural frequency shifts occur during the random excitation when compared to the harmonic test. During the

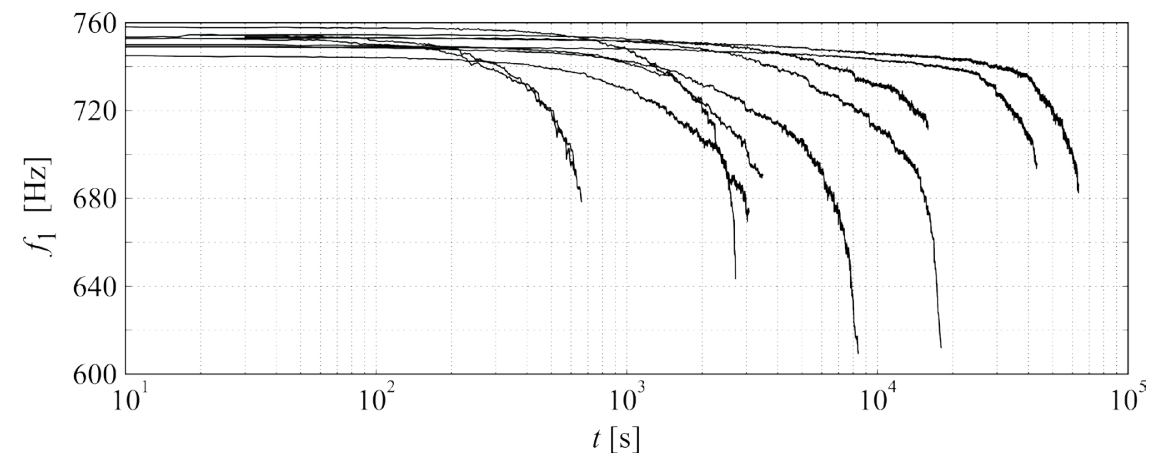

Fig. 9. Natural frequency drop during the random-excitation test 
harmonic test a drop of natural frequency occurred just before the crack propagated through the whole cross-section. For that case the real-time control was not sufficiently reliable and the fatigue failure was declared at this point. On the other hand, when the excitation is a random process, the fatigue crack propagates continuously until the full cross-section crack is reached. Since many authors [5] and [21] assume that frequency shift relates to the accumulated fatigue damage the fatigue failure of the specimen under random excitation was declared when a certain value of the natural frequency shift was observed. The critical natural frequency shift for the final fatigue failure at the random-excitation test was $23.9 \mathrm{~Hz}$, i.e. the average value of the total frequency shift, measured for the specimens at the harmonic tests (see Table 1).

\subsection{Numerical Model}

The vibration fatigue life of the single tested specimen was obtained in the customly developed Vibrationfatigue plug-in for the Catia analysis environment [22]. The FEM model consisted of 15600 10-node tetrahedral solid elements. The size of the elements in the fatigue zone was approximately $1.5 \mathrm{~mm}$, which provided reliable results of stress responses used for the fatigue life estimation. As shown in [9], the measured and numerically obtained stress distributions in the fatigue zone were in a good agreement. For the fatigue life estimation the material parameters from harmonic test along with the calculated nodal values of equivalent stress from modal analysis were used. Additionally, the initial damping loss factors (Table 2) were used to predict the $S_{\sigma_{e q}}(\omega)$, defined in Eq. (10). In Fig. 10 the fatigue life estimation for specimen $\mathrm{S} 12$ is visualized. In order to reduce the calculation time, the fatigue life calculation was performed for the nodes in the fatigue zone, only.

The comparison between the estimated and actual fatigue lives of the specimens for the case of random excitation is shown in Fig. 11. From this figure one can notice, that the numerical analysis estimated reasonably shorter fatigue lives compared to the actual fatigue lives. This deviation originates from the damping loss factor, proposed in the numerical calculation, which was measured for the intact specimen at a low excitation level. From the discussion regarding the results of the harmonic test the damping loss factor increases with the accumulated fatigue damage and with the higher excitation level (due to the type of specimen fixation). In any case, the fatigue life estimation gives conservative prediction when the initial damping loss factor is used.

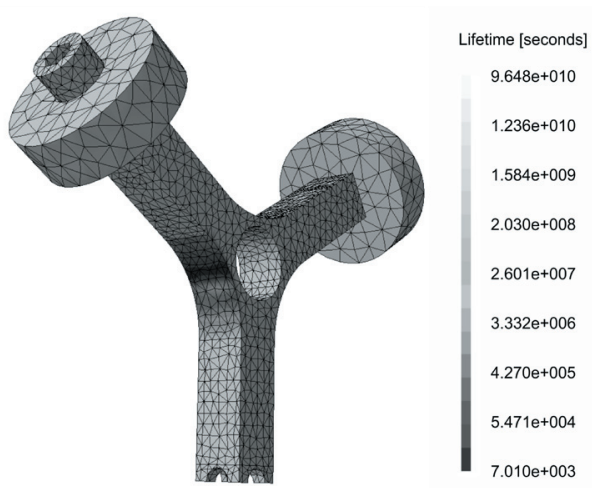

Fig. 10. Expected fatigue life for specimen S12

\subsection{Comparison of the Experimental and Numerical Results}

Additionally, for the tested series of specimens, the effective damping loss factor was obtained with numerical minimization that gives a minimal sum of deviations between actual $T_{a c t}$ and estimated $T_{e s t}$ fatigue lives:

$$
\Delta T(\eta)=\sum_{i}\left(\log _{10}\left(T_{a c t, i}\right)-\log _{10}\left(T_{e s t, i}(\eta)\right)\right)^{2} .
$$

The numerical optimization was performed with the simplex method. The value of effective damping loss factor was identified as $9.594 \times 10^{-4}$. The comparison between experiment and fatigue life estimation using the updated value of damping loss factor is shown in Fig. 12.

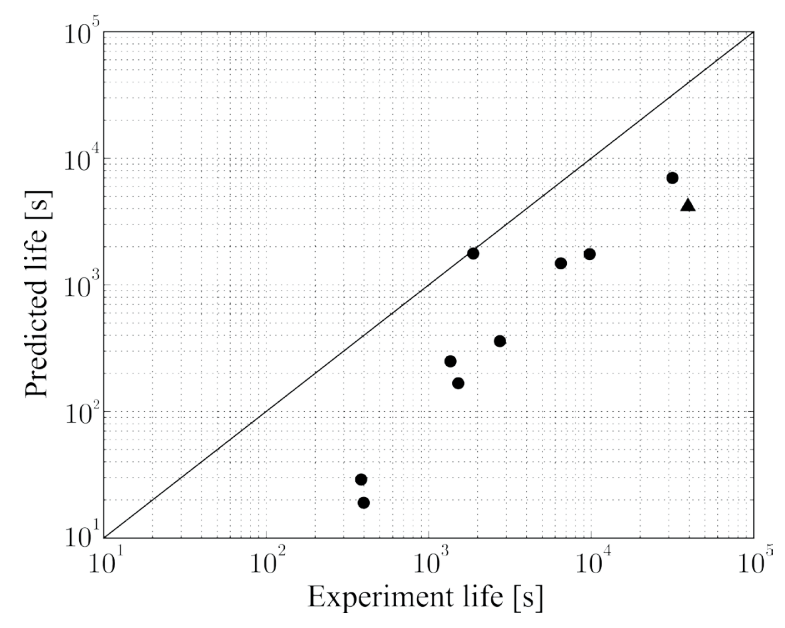

Fig. 11. Comparison of random excitation experiment with numerical prediction using the initial damping loss factors

From the presented comparison one can conclude, that the damping loss factor greatly influences the 
fatigue life estimation. Observing the specimen S11 (marked with $\boldsymbol{\Delta}$ in Figs. 11 and 12): if the value of damping loss factor is increased by factor of 2.35 the predicted fatigue life increases by a factor of 11.3.

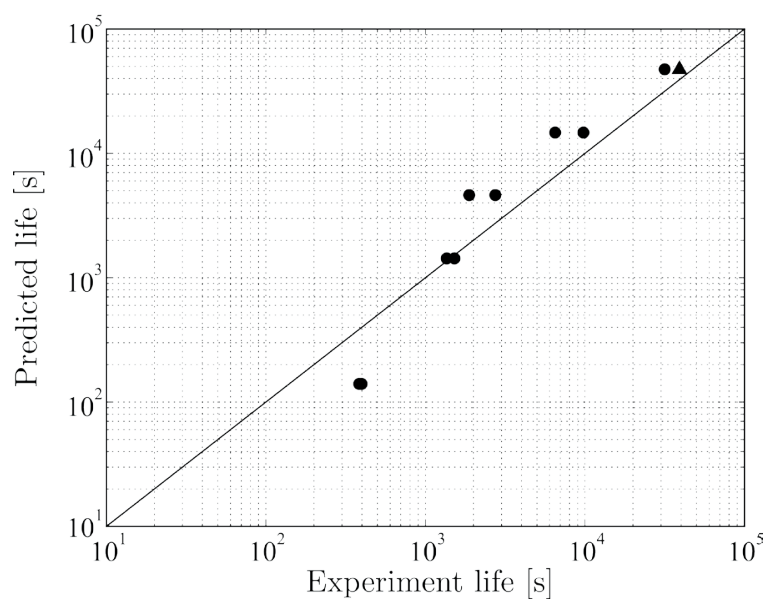

Fig. 12. Comparison of random excitation experiment with numerical prediction using the updated damping loss factor

The presented numerical and experimental results clearly show that uncertainties of the fatigue-life estimation rise from the inherent changes of system's modal properties during the damage accumulation and consequential crack propagation. In order to bring the numerical fatigue-life estimation closer to the real-life vibration fatigue phenomenon, the need for further and more comprehensive research is obvious. Due to this reason, the research presented here serves as a basis for future research work.

\section{CONCLUSIONS}

In this study an accurate examination of the vibration fatigue phenomenon is performed. The vibrational fatigue was for the case of Y-shaped specimens experimentally performed with a harmonic and random base-excitation. The comparison between experimental and numerical results was also given. Several conclusions can be drawn, which are stated in the following paragraphs.

The presented experiment with the harmonic excitation has several advantages. Firstly, with the described experimental methodology it is possible to perform a fast fatigue test with a simultaneous tracking of the changes of the modal parameters. Additionally, an indirect measurement of the stress load, based only on measurements of the excitation and response acceleration, greatly shortens the specimen preparation time and additionally improves the reliability of the stress measurement during the whole accelerated fatigue test compared to the traditional strain-gauge method. In its present state, the experimental setup is used for the rapid acquisition of a material's fatigue parameters, since a highcycle accelerated fatigue test of $2 \times 10^{7}$ load cycles is achieved in approximately 7 hours.

The fatigue test with random excitation diminishes the influence of the natural frequency shift on the response amplitude, as long as the acceleration profile with constant acceleration PSD is applied. On the other hand, the effect of the damping increase, which occurs with accumulated damage and at higher excitation levels, is present also in the randomexcitation testing. By comparing the experimental and numerical results the damping increase assures the conservative fatigue life prediction. Therefore, by adopting the initial damping loss factor, obtained with experimental modal analysis at low excitation levels, the fatigue life estimation is always on the conservative side.

\section{REFERENCES}

[1] Lee, Y.-L., Pan, J., Hathaway, R.B., Barkey, M.E. (2005). Fatigue Testing and Analysis: Theory and Practice. Elsevier, Oxford.

[2] Kranjc, T., Slavič, J., Boltežar, M. (2013). The mass normalization of the displacement and strain mode shapes in a strain experimental modal analysis using the mass-change strategy. Journal of Sound and Vibration, vol. 332, no. 26, p. 6968-6981, DOI:10.1016/j. jsv.2013.08.015.

[3] Maia, N.M.M., Silva, J.M.M. (1997). Theoretical and Experimental Modal Analysis. Research Studies Press Ltd., Baldock, Hertfordshire.

[4] Doebling, S.W., Farrar, C.R., Prime, M.B., Shevitz, D.W. (1996). Damage Identification and Health Monitoring of Structural and Mechanical Systems from Changes in Their Vibration Characteristics: A Literature Review, Technical Report. Los Alamos, New Mexico.

[5] Dirlik, T. (1985). Application of Computers in Fatigue Analysis. PhD thesis, University of Warwick, Warwick.

[6] Béliveau, J.-G., Vigneron, F.R., Soucy, Y., Draisey, S. (1985). Modal parameter estimation from base excitation. Journal of Sound and Vibration, vol. 107, no. 3, p. 435-449, DOI:10.1016/S0022-460X(86)80117-1.

[7] Shang, D.G., Barkey, M.E., Wang, Y., Lim, T.C. (2003). Effect of fatigue damage on the dynamic response frequency of spot-welded joints. International Journal of Fatigue, vol. 25, no. 4, p. 311-316, DOI:10.1016/ S0142-1123(02)00140-8.

[8] Colakoglu, M., Jerina, K.L. (2003). Material damping in 6061-T6511 aluminium to assess fatigue damage. Fatigue and Fracture of Engineering Materials and 
Structures, vol. 26, no. 1, p. 79-84, DOI:10.1046/ j.1460-2695.2003.00603.x.

[9] Čermelj, P., Boltežar, M. (2006). Modelling localised nonlinearities using the harmonic nonlinear super model. Journal of Sound and Vibration, vol. 298, no. 4-5, p. 1099-1112, DOI:10.1016/j.jsv.2006.06.042.

[10] Česnik, M., Slavič, J., Boltežar, M. (2012). Uninterrupted and accelerated vibrational fatigue testing with simultaneous monitoring of the natural frequency and damping. Journal of Sound and Vibration, vol. 331, no. 24, p. 5370-5382, DOI:10.1016/j.jsv.2012.06.022.

[11] Benasciutti, D. (2004). Fatigue Analysis of Random Loadings. $\mathrm{PhD}$ thesis, University of Ferrara, Ferrara.

[12] Pagnacco, E., Lambert, S., Khalij, L., Rade, D.A. (2012). Design optimisation of linear structures subjected to dynamic random loads with respect to fatigue life. International Journal of Fatigue, vol. 43, p. 168-177, DOI:10.1016/j.ijfatigue.2012.04.001.

[13] Paulus, M., Dasgupta, A. (2012). Semi-empirical life model of a cantilevered beam subject to random vibration. International Journal of Fatigue, vol. 45, p. 82-90, DOI:10.1016/j.ijfatigue.2012.06.008.

[14] Han, S.-H., An, D.-G., Kwak, S.-J., Kang, K.-W. (2013). Vibration fatigue analysis for multi-point spotwelded joints based on frequency response changes due to fatigue damage accumulation. International Journal of Fatigue, vol. 48, p. 170-177, DOI:10.1016/j. ijfatigue.2012.10.017.

[15] Chen, Y.S., Wang, C.S., Yang, Y.J. (2008). Combining vibration test with finite element analysis for the fatigue life estimation of PBGA components. Microelectronics
Reliability, vol. 48, no. 4, p. 638-644, DOI:10.1016/j. microrel.2007.11.006.

[16] Yu, D., Al-Yafawi, A., Nguyen, T.T., Park, S., Chung, S. (2011). High-cycle fatigue life prediction for $\mathrm{Pb}$-free BGA under random vibration loading. Microelectronics Reliability, vol. 51, no. 3, p. 649-656, DOI:10.1016/j. microrel.2010.10.003.

[17] Ewins, D.J. (2000). Modal Testing: Theory, Practice and Application. Research Studies Press Ltd., Baldock, Hertfordshire.

[18] Mršnik, M., Slavič, J., Boltežar, M. (2013). Frequency-domain methods for a vibration-fatiguelife estimation - Application to real data. International Journal of Fatigue, vol. 47, p. 8-17, DOI:10.1016/j. ijfatigue.2012.07.005.

[19] Pitoiset, X., Preumont, A. (2000). Spectral methods for multiaxial random fatigue analysis of metallic structures. International Journal of Fatigue, vol. 22, no. 7, p. 541-550, DOI:10.1016/S0142-1123(00)000384.

[20] US Department of the Air Force (2008). MIL-STD-810, Military standard environmental test methods for aerospace and ground equipment, revision G, Method 514.6: Vibration, United States Department of Defense, Arlington.

[21] Wang, R.J., Shang, D.G. (2009). Fatigue life prediction based on natural frequency changes for spot welds under random loading. International Journal of Fatigue, vol. 31, no. 2, p. 361-366, DOI:10.1016/j.ijfatigue. 2008.08.001.

[22] Dasault systemes (2014). from http://www.3ds.com/ products-services/catia, accessed on 2014- 04-09. 